\title{
Cognitive Engineering for Technology in Mental Health Care and Rehabilitation
}

\author{
Willem-Paul Brinkman \\ Delft University of \\ Technology \\ w.p.brinkman@tudelft.nl
}

\author{
Gavin Doherty \\ Trinity College Dublin \\ gavin.doherty@tcd.ie
}

\author{
Alessandra Gorini, Andrea \\ Gaggioli \\ Istituto Auxologico Italiano \\ Alessandra.gorini@gmail.com, \\ andrea.gaggioli@unicatt.it
}

\author{
Mark Neerincx \\ TNO Human \\ Factors/Delft University \\ of Technology \\ mark.neerincx@tno.nl
}

\begin{abstract}
The use of technology, such as virtual reality, electronic diaries, multimedia, brain computing and computer games, to support the care and rehabilitation of patients affected by mental disorders is a relatively new and advancing research area. In this workshop, researchers, developers and mental health professionals will present and discuss their latest work, with a focus on cognitive, emotional and ergonomic aspects on issues as acceptance, usage, experience and accessibility of these innovative technologies.
\end{abstract}

\section{Keywords}

Mental health, virtual reality, care, rehabilitation, resilience, support technology, psychotherapy, cognitive ergonomics. cybertherapy.

\section{INTRODUCTION}

The use of technology to support diagnostics and treatments in mental health care and rehabilitation is increasing. Research efforts in the area have also begun to consider strengthening resilience against mental stress and enhancing overall mental wellness. Although considerable attention has been devoted to evaluating the effectiveness of these technologies, little attention has been given to the cognitive ergonomic aspects of using these technologies, from the perspective of either the mental healthcare professional or of the patient. New innovative, efficient, and accessible technology is needed for safe care and rehabilitation. Technologies such as physiological measurement or visualisation technology should be applied in a manner that would create easy to use, acceptable and accessible systems. A cognitive engineering approach with its traditional focus on users, (in this case patients and mental healthcare professionals), could help to improve the interaction between the users and the system considering technological innovations, cognitive ergonomic knowledge and situational demands. Still the question is: are there any existing lessons, guidelines or methods from cognitive engineering that could be used? Or does this domain need its own methods and set of guidelines? In a still relevant SWOT analysis of the field of VR rehabilitation Rizzo and Kim (2005) identified limitations in the areas of interfaces and display technology and stressed the continuous need for usercentred design and evaluation methods. They also mentioned the importance of developing strong multidisciplinary collaboration between engineers and clinicians. Such collaboration should help to adress and overcome objections clinicians have raised regarding the use of technological innovations and allay fears that doing so will affect both the patient-therapist relationship, and the therapist's ability to control care and rehabilitation. In addition, technology might also provide new ways to deal with stigmas that are associated with seeking help for mental health problems. For example the use of technology could help reduce the number of visits to a clinic, or facilitate treatment over the internet. Making the treatment or care more accessible to patients could also entail enhancing the level of engagement or persuasiveness of the technology, especially for technologies such as virtual coaches or educational games. Another key factor, for example in the case of tele-health or virtual reality applications, is the feeling of presence, the subjective experience of being there. Failure to establish this might cause dropouts or a lack of clinical improvement.

Conducting research in this domain also needs to address the ethical constraints for empirical studies that involve patients. In particular to conduct research with mental health patients, researchers should consider the ability of these patients to give research consent disclosure and the way in which this should be obtained. This also relates to the more fundamental question pertaining to the issue of the appropriate research methodology in this domain. This especially in relation to the issue of when to involve patients and the possibility of using an alternative, less vulnerable subject group and how to generalise findings for a patient group. Understanding how different patient factors influence the effectiveness of treatment in a given context is a prerequisite for this.

\section{OBJECTIVE}

The workshop seeks to attract researchers, designers, and practitioners interested in understanding good design and engineering in the domain of mental health. Workshop participants could come from a variety of backgrounds such as computer science, psychology, design, and psychiatry. The main objective of the workshop is to strengthen this growing community within the domain, thereby allowing a lively exchange of ideas with the aim of supporting the research of the 
workshop members, as well as working towards the publication of the special issue of the Journal of CyberTherapy \& Rehabilitation.

\section{WORKSHOP FOCUS}

Workshops that focus on cognitive ergonomics in relation to technology in health care are not new; take for example HCI2008 workshop on the HCI for Medicine and Health Care ${ }^{12}$, the CHI 2009 workshop on Evaluating new Interactions in Healthcare, and the symposium Usability \& Human-Computer Interaction for Medicine and Health Care (Holzinger, 2007). Few, however, focus specifically on the domain of mental health such as CHI 2008 Workshop on Technology in Mental Health ${ }^{1314}$. This workshop will continue this tradition and focus on the technology used in clinical areas such as anxiety disorder, addictions, cognitive rehabilitation, eating disorders, motor rehabilitation, obesity, pain management, personality disorder and autism. Possible topics in this area include, but are not limited to:

- Bio/feedback/physiological measurement

- Simulation/augmentation and presence

- Virtual and augmented reality

- Brain computing interfaces

- Wearable technology

- Internet based technology, tele-health and videoconferencing

- Mobile technology, including mobile diary systems

- Display technology (CAVE, HMD, Projection systems, etc)

- Therapeutic computer games

- Educational and persuasive technology, including psycho-education systems

- Technology support to enhance persons' resilience in stressful situations

\section{WORKSHOP FORMAT}

This is a full-day workshop. Workshop members will present their work followed by an in-depth discussion. Participants are encouraged to demonstrate the support technology that they might have developed, used, or studied. In the afternoon, participants will also discuss opportunities for the special journal issue, ways of collaboration and new research questions. The workshop will also be supported by a website. The draft position papers will be posted here in advance, and participants' slides will also be made available on the website before the workshop. After the workshop, position papers and minutes from the workshop

\footnotetext{
12 http://hci4all.at/HCI4MED08.html

${ }^{13} \mathrm{http}: / /$ healthcare-interactions.blogspot.com

${ }^{14}$ https://www.cs.tcd.ie/TIMH/
}

discussions will be published in the proceedings of the workshop.

\section{PROGRAM COMMITTEE}

- Mariano Alcañiz, Universidad Politécnica de Valencia, Spain

- Cristina Botella, Universitat Jaume I, Spain.

- Paul Emmelkamp, University of Amsterdam, The Netherlands.

- Robert Hubal, RTI International, USA

- Charles van der Mast, Delft University of Technology, The Netherlands

- Evelyne Klinger, Arts et Métiers ParisTech LAMPA, France

- Paul Kizakevich, RTI International, USA

- Giuseppe Riva, Università Cattolica del Sacro Cuore, Italy

- James Spira, University of California San Diego, USA

- Daniel Thalmann, Swiss Federal Institute of Technology, Switzerland

- Arnold Vermeeren, Delft University of Technology, The Netherlands

- $\quad$ Eric Vermetten, Military Mental Health Research Center, Central Military Hospital, Utrecht, The Netherlands / Rudolf Magnus Institute of Neuroscience, University Medical Center, Utrecht, The Netherlands

- Valentijn Visch, Delft University of Technology, The Netherlands.

- Brenda Wiederhold, Interactive Media Institute, USA

\section{REFERENCES}

Holzinger, A. (2007). HCI and usability for medicine and Health Care. Springer Lecture Notes in Computer Science 4799.

Rizzo, A., \& Kim, G. J. (2005). A SWOT analysis of the field of virtual reality rehabilitation and therapy. Presence-Teleoperators and Virtual Environments, 14(2), 119-146. 